\title{
Hybrid iterative methods for two asymptotically nonexpan- sive semigroups in Hilbert spaces
}

\section{Issara Inchan}

Department of Mathematics, Uttaradit Rajabhat University, Uttaradit, Thailand.

\begin{abstract}
The main objective of this work is to modify two hybrid projection algorithm. First, we prove the strongly convergence to common fixed points of a sequence $\left\{x_{n}\right\}$ generated by the hybrid projection algorithm of two asymptotically nonexpansive mappings, second, we prove the strongly convergence of a sequence $\left\{x_{n}\right\}$ generated by the hybrid projection algorithm of two asymptotically nonexpansive semigroups. Our main results extend and improve the results of Dong et al. [Q.-L. Dong, S. N. He, Y. J. Cho, Fixed Point Theory Appl., 2015 (2015), 12 pages].
\end{abstract}

Keywords: Asymptotically nonexpansive mappings, asymptotically nonexpansive semigroup, fixed point. 2010 MSC: 46C05, 47D03, 47H09, 47H10, 47H20.

(C)2019 All rights reserved.

\section{Introduction}

Let $\mathrm{H}$ be a real Hilbert space, $\mathrm{C}$ a nonempty closed convex subset of $\mathrm{H}$ and $\mathrm{T}: \mathrm{C} \rightarrow \mathrm{C}$ a mapping. Recall that a self-mapping $f$ of $C$ is a contraction if $\|f(x)-f(y)\| \leqslant \alpha\|x-y\|$ for some $\alpha \in(0,1)$ and $T$ is a nonexpansive if $\|T x-T y\| \leqslant\|x-y\|$ for all $x, y \in C$, and $T$ is asymptotically nonexpansive [2] if there exists a sequence $\left\{k_{n}\right\}$ with $k_{n} \geqslant 1$ for all $n$ and $\lim _{n \rightarrow \infty} k_{n}=1$ and such that $\left\|T^{n} x-T^{n} y\right\| \leqslant k_{n}\|x-y\|$ for all $n \geqslant 1$ and $x, y \in C$. A point $x \in C$ is a fixed point of $T$ provided $T x=x$. Denote by Fix(T) the set of fixed points of $\mathrm{T}$, that is, $\operatorname{Fix}(\mathrm{T})=\{x \in \mathrm{C}: T x=x\}$.

Recall also that a one-parameter family $\mathcal{T}=\{\mathrm{T}(\mathrm{t}) \mid 0 \leqslant \mathrm{t}<\infty\}$ of self-mappings of a nonempty closed convex subset $\mathrm{C}$ of a Hilbert space $\mathrm{H}$ is said to be a (continuous) Lipschitzian semigroup on $\mathrm{C}$ (see, e.g., [12]) if the following conditions are satisfied:

(i) $\mathrm{T}(0) \mathrm{x}=\mathrm{x}, \quad \mathrm{x} \in \mathrm{C}$;

(ii) $\mathrm{T}(\mathrm{s}+\mathrm{t})(\mathrm{x})=\mathrm{T}(\mathrm{s}) \mathrm{T}(\mathrm{t}), \quad \mathrm{s}, \mathrm{t} \geqslant 0, x \in \mathrm{C}$;

(iii) for each $x \in C$, the map $t \mapsto T(t) x$ is continuous on $[0, \infty)$;

Email address: peissara@uru.ac.th (Issara Inchan)

doi: $10.22436 /$ jnsa.012.10.01

Received: 2018-07-18 Revised: 2019-04-23 Accepted: 2019-05-10 
(iv) there exists a bounded measurable function $L:[0, \infty) \rightarrow[0, \infty)$ such that, for each $t>0$

$$
\|T(t) x-T(t) y\| \leqslant L_{t}\|x-y\|, \quad x, y \in C .
$$

A Lipschitzian semigroup $\mathcal{T}$ is called nonexpansive (or a contraction semigroup) if $L_{t}=1$ for all $t>0$, and asymptotically nonexpansive semigroup if $\limsup _{t \rightarrow \infty} L_{t} \leqslant 1$, respectively. We use $\operatorname{Fix}(\mathcal{T})$ to denote the common fixed point set of the semigroup, that is $\operatorname{Fix}(\mathcal{T})=\{x \in C: T(t) x=x, t>0\}$.

Fixed point iteration processes for nonexpansive mappings and asymptotically nonexpansive mappings in Hilbert spaces and Banach spaces including Mann and Ishikawa iteration processes have been studied extensively by many authors to solve nonlinear operator equations as well as variational inequalities, see [4, 7, 9-11]. However, Mann and Ishikawa iterations processes have only weak convergence even in Hilbert space, see [5, 11].

Very recently, Takahashi et al. [11] proved the following strong convergence theorems by the hybrid method for nonexpansive mappings and nonexpansive semigroup in Hilbert space.

Theorem 1.1 ([11]). Let $\mathrm{H}$ be a Hilbert space and $\mathrm{C}$ be a nonempty closed convex subset of $\mathrm{H}$. Let $\mathrm{T}$ be a nonexpansive mapping of $\mathrm{C}$ into $\mathrm{H}$ such that $\mathrm{F}(\mathrm{T}) \neq \emptyset$ and let $\mathrm{x}_{0} \in \mathrm{H}$. For $\mathrm{C}_{1}=\mathrm{C}$ and $\mathrm{u}_{1}=\mathrm{P}_{\mathrm{C}_{1}} \mathrm{x}_{0}$, define a sequence $\left\{\mathrm{u}_{\mathrm{n}}\right\}$ of $\mathrm{C}$ as follows:

$$
\left\{\begin{array}{l}
y_{n}=\alpha_{n} u_{n}+\left(1-\alpha_{n}\right) T u_{n}, \\
C_{n+1}=\left\{z \in C_{n}:\left\|y_{n}-z\right\| \leqslant\left\|u_{n}-z\right\|\right\} \\
u_{n+1}=P_{C_{n+1}} x_{0}, n \in \mathbb{N}
\end{array}\right.
$$

where $0 \leqslant \alpha_{n} \leqslant a<1$ for all $n \in \mathbb{N}$. Then $\left\{u_{n}\right\}$ converges strongly to $z_{0}=P_{F(T)} x_{0}$.

In 2008, Inchan and Plubtieng [3] modified Ishikawa iteration process for two asymptotically nonexpansive mappings, for $C$ is a nonempty closed convex subset of a Hilbert space $H$, let $x_{0} \in C$. For $C_{1}=C$ and $x_{1}=P_{C_{1}}$, define $\left\{x_{n}\right\}$ as follows:

$$
\left\{\begin{array}{l}
y_{n}=\alpha_{n} x_{n}+\left(1-\alpha_{n}\right) T^{n} z_{n} \\
z_{n}=\beta_{n} x_{n}+\left(1-\beta_{n}\right) S^{n} x_{n} \\
C_{n+1}=\left\{z \in C_{n}:\left\|y_{n}-z\right\|^{2} \leqslant\left\|x_{n}-z\right\|^{2}+\theta_{n}\right\} \\
x_{n+1}=P_{C_{n+1}} x_{0}, n \in \mathbb{N},
\end{array}\right.
$$

where $\theta_{n}=\left(1-\alpha_{n}\right)\left[\left(t_{n}^{2}-1\right)+\left(1-\beta_{n}\right) t_{n}^{2}\left(s_{n}^{2}-1\right)\right](\operatorname{diamC})^{2} \rightarrow 0$ as $n \rightarrow \infty$ and $0 \leqslant \alpha_{n} \leqslant a<1$ and $0<\mathrm{b} \leqslant \beta_{\mathrm{n}} \leqslant \mathrm{c}<1$ for all $\mathrm{n} \in \mathbb{N}$. Then the sequence $\left\{x_{n}\right\}$ converges strongly to common fixed points of two asymptotically nonexpansive mappings.

In 2015, Dong et al. [1], introduced a hybrid algorithm. Let $\mathrm{T}$ and $\mathrm{S}$ be two nonexpansive mappings into itself such that $F(T) \cap F(S) \neq \emptyset$, the sequence generated as follows:

$$
\left\{\begin{array}{l}
x_{0} \in C, \\
y_{n}=\alpha_{n} x_{n}+\left(1-\alpha_{n}\right) T x_{n}, \\
z_{n}=\beta_{n}\left[\gamma_{n} y_{n}+\left(1-\gamma_{n}\right) x_{n}\right]+\left(1-\beta_{n}\right) S y_{n}, \\
C_{n}=\left\{z \in C: \sigma\left\|z_{n}-z\right\|^{2}+(1-\sigma)\left\|y_{n}-z\right\|^{2} \leqslant\left\|x_{n}-z\right\|^{2}\right\}, \\
Q_{n}=\left\{z \in C:\left\langle x_{n}-z, x_{n}-x_{0}\right\rangle \leqslant 0\right\}, \\
x_{n+1}=P_{C_{n} \cap Q_{n}} x_{0}, n \geqslant 0,
\end{array}\right.
$$

for each $n \geqslant 0$, where $\alpha_{n}, \beta_{n} \in[0,1], \delta \in[0,1), \gamma_{n} \in[0,1], \sigma \in(0,1)$. Then proved that $\left\{x_{n}\right\}$ converges in norm to $\mathrm{P}_{\mathrm{F}(\mathrm{T}) \cap \mathrm{F}(\mathrm{S})} \mathrm{x}_{0}$. 
Inspired and motivated by above, the purpose of this paper is to extend the results of Dong et al. [1] for $S$ and $T$ are two asymptotically nonexpansive mappings then we consider

$$
\left\{\begin{array}{l}
x_{0} \in C=C_{1}, \quad x_{1}=P_{C_{1}} x_{0}, \\
y_{n}=\alpha_{n} x_{n}+\left(1-\alpha_{n}\right) T^{n} x_{n}, \\
z_{n}=\beta_{n}\left[\gamma_{n} y_{n}+\left(1-\gamma_{n}\right) x_{n}\right]+\left(1-\beta_{n}\right) S^{n} y_{n}, \\
C_{n+1}=\left\{z \in C_{n}:\left\|z_{n}-z\right\|^{2}+\left\|y_{n}-z\right\|^{2} \leqslant 2\left\|x_{n}-z\right\|^{2}+\theta_{n}\right\} \\
x_{n+1}=P_{C_{n+1}} x_{0}, n \geqslant 0,
\end{array}\right.
$$

where $\alpha_{n}, \beta_{n}, \gamma_{n} \in[0,1]$ and

$$
\theta_{n}=\left[\left(1-\beta_{n}\right)\left(s_{n}^{2}-1\right)+\left(1-\alpha_{n}\right)\left(t_{n}^{2}-1\right)+\left(1-\beta_{n}\right) s_{n}^{2}\left(1-\aleph_{n}\right)\left(t_{n}^{2}-1\right)\right](\operatorname{diamC})^{2} \rightarrow 0,
$$

as $n \rightarrow \infty$ (here $t_{n} \rightarrow 1$ and $s_{n} \rightarrow 1$ as $n \rightarrow \infty$ ). Then, under some contral conditions we show the strongly convergence of $\left\{x_{n}\right\}$.

\section{Preliminaries}

In this section, we collect and give some useful lemmas that will be used for our main result in the next section.

Lemma 2.1. Let $\mathrm{H}$ be a real Hilbert space, then the following hold:

(i) $\|x+y\|^{2} \leqslant\|x\|^{2}+2\langle x, y\rangle+\|y\|^{2}, \forall x, y \in H$;

(ii) $\|\mathrm{t} x+(1-\mathrm{t}) \mathrm{y}\|^{2}=\mathrm{t}\|\mathrm{x}\|^{2}+(1-\mathrm{t})\|\mathrm{y}\|^{2}-\mathrm{t}(1-\mathrm{t})\|\mathrm{x}-\mathrm{y}\|^{2}, \mathrm{t} \in[0,1], \quad \forall x, y \in \mathrm{H}$.

Lemma 2.2 ([5]). Let $\mathrm{C}$ be a nonempty bounded closed convex subset of real Hilbert space $\mathrm{H}$ and let

$$
\mathcal{T}:=\{\mathrm{T}(\mathrm{s}): 0 \leqslant \mathrm{~s}<\infty\},
$$

an asymptotically nonexpansive semigroup on $\mathrm{C}$. If $\left\{\mathrm{x}_{\mathrm{n}}\right\}$ is a sequence in $\mathrm{C}$ satisfying the properties

(i) $x_{n} \rightarrow z$;

(ii) $\limsup _{t \rightarrow \infty} \limsup _{n \rightarrow \infty}\left\|\mathrm{T}(\mathrm{t}) \mathrm{x}_{\mathrm{n}}-\mathrm{x}_{\mathrm{n}}\right\|=0$;

then $z \in \operatorname{Fix}(\mathcal{T})$.

Lemma 2.3 ([5]). Let $\mathrm{C}$ be a nonempty bounded closed convex subset of real Hilbert space $\mathrm{H}$ and let

$$
\mathcal{T}:=\{\mathrm{T}(\mathrm{s}): 0 \leqslant s<\infty\},
$$

an asymptotically nonexpansive semigroup on $\mathrm{C}$. Then for any $\mathrm{u} \geqslant 0$,

$$
\limsup _{\mathfrak{u} \rightarrow \infty} \limsup _{t \rightarrow \infty} \sup _{x \in C}\left\|\frac{1}{t} \int_{0}^{t} T(s) x d s-T(u)\left(\frac{1}{t} \int_{0}^{t} T(s) x d s\right)\right\|=0 .
$$

Lemma 2.4 ([6]). Let $\mathrm{T}$ be an asymptotically nonexpansive mapping defined on a bounded convex subset $\mathrm{C}$ of a Hilbert space $\mathrm{H}$. If $\left\{x_{n}\right\}$ is a sequence in $C$ such that $x_{n} \rightarrow x$ and $T x_{n}-x_{n} \rightarrow 0$, then $x \in F(T)$.

Lemma 2.5 ([8]). Let $\mathrm{C}$ be a nonempty closed convex subset of $\mathrm{H}$. Let $\left\{x_{\mathrm{n}}\right\}$ be a sequence in $\mathrm{H}$ and $\mathrm{u} \in \mathrm{H}$. Let $\mathrm{q}=\mathrm{P}_{\mathrm{C}} \mathrm{u}$. If $\left\{\mathrm{x}_{\mathrm{n}}\right\}$ is such that $\omega_{w}\left(x_{n}\right) \subset \mathrm{C}$ and satisfies the condition

$$
\left\|x_{n}-u\right\| \leqslant\|u-q\|,
$$

for all $\mathrm{n} \geqslant 1$, then $\mathrm{x}_{\mathrm{n}} \rightarrow \mathrm{q}$. 


\section{Main result}

In this section we introduce two theorems. We first prove the strong convergence theorem of modified the hybrid method of of asymptotically nonexpansive mappings into $P_{F(T) \cap F(S)} x_{0}$. As the second part, we prove the strong convergence of modified the hybrid method of asymptotically nonexpansive semigroups into $P_{\mathfrak{J}} x_{0}$.

\subsection{Strong convergence theorem of asymptotically nonexpansive mappings}

In this section, we prove the strong convergence theorem of the algorithm (1.1) into $\mathrm{P}_{\mathrm{F}(\mathrm{T}) \cap \mathrm{F}(\mathrm{S})} \mathrm{x}_{0}$.

Theorem 3.1. Let $\mathrm{C}$ be a nonempty closed convex subset of a Hilbert space $\mathrm{H}$ and $\mathrm{T}, \mathrm{S}: \mathrm{C} \rightarrow \mathrm{C}$ be two asymptotically nonexpansive mappings with the sequences $\left\{\mathrm{t}_{\mathrm{n}}\right\}$ and $\left\{\mathrm{s}_{\mathrm{n}}\right\}$, respectively, such that $\mathrm{F}(\mathrm{T}) \cap \mathrm{F}(\mathrm{S}) \neq \emptyset$. Assume that $\left\{\alpha_{n}\right\},\left\{\beta_{n}\right\}$ and $\left\{\gamma_{n}\right\}$ are the sequences in $[0,1]$ such that $\alpha_{n}, \beta_{n} \leqslant 1-\delta$ for some $\delta \in(0,1]$. Then the sequence $\left\{x_{n}\right\}$ generated by (1.1) converges in norm to $\mathrm{P}_{\mathrm{F}(\mathrm{T}) \cap \mathrm{F}(\mathrm{S})} \mathrm{x}_{0}$.

Proof. Putting $t_{\infty} \sup \left\{t_{n}: n \geqslant 1\right\}<\infty$ and $s_{\infty} \sup \left\{s_{n}: n \geqslant 1\right\}<\infty$. We first show by induction that $F(T) \cap F(S) \subseteq C_{n}$ for all $n \in \mathbb{N}$. It is obvious that $F(T) \cap F(S) \subseteq C_{1}$. Suppose that $F(T) \cap F(S) \subseteq C_{k}$ for each $k \in \mathbb{N}$. Let $u \in F(T) \cap F(S) \subseteq C_{k}$, then from Lemma 2.1, we have

$$
\begin{aligned}
\left\|y_{k}-u\right\|^{2} & =\left\|\alpha_{k} x_{k}+\left(1-\alpha_{k}\right) T^{k} x_{k}-u\right\|^{2} \\
& =\left\|\alpha_{k}\left(x_{k}-u\right)+\left(1-\alpha_{k}\right)\left(T^{k} x_{k}-u\right)\right\|^{2} \\
& =\alpha_{k}\left\|x_{k}-u\right\|^{2}+\left(1-\alpha_{k}\right)\left\|T^{k} x_{k}-u\right\|^{2}-\alpha_{k}\left(1-\alpha_{k}\right)\left\|x_{k}-T^{k} x_{k}\right\|^{2} \\
& \leqslant \alpha_{k}\left\|x_{k}-u\right\|^{2}+\left(1-\alpha_{k}\right)\left\|T^{k} x_{k}-u\right\|^{2} \\
& \leqslant \alpha_{k}\left\|x_{k}-u\right\|^{2}+\left(1-\alpha_{k}\right) t_{k}^{2}\left\|x_{k}-u\right\|^{2} \\
& =\left\|x_{k}-u\right\|^{2}-\left\|x_{k}-u\right\|^{2}+\alpha_{k}\left\|x_{k}-u\right\|^{2}+\left(1-\alpha_{k}\right) t_{k}^{2}\left\|x_{k}-u\right\|^{2} \\
& =\left\|x_{k}-u\right\|^{2}-\left(1-\alpha_{k}\right)\left\|x_{k}-u\right\|^{2}+\left(1-\alpha_{k}\right) t_{k}^{2}\left\|x_{k}-u\right\|^{2} \\
& =\left\|x_{k}-u\right\|^{2}+\left(1-\alpha_{k}\right)\left(t_{k}^{2}-1\right)\left\|x_{k}-u\right\|^{2} .
\end{aligned}
$$

Similarly, we note that from Lemma 2.1 and (3.1), we have

$$
\begin{aligned}
\left\|z_{k}-u\right\|^{2}= & \left\|\beta_{k}\left[\gamma_{k} y_{k}+\left(1-\gamma_{k}\right) x_{k}\right]+\left(1-\beta_{k}\right) S^{k} y_{k}-u\right\|^{2} \\
= & \left\|\beta_{k}\left(\left[\gamma_{k} y_{k}+\left(1-\gamma_{k}\right) x_{k}\right]-u\right)+\left(1-\beta_{k}\right)\left(S^{k} y_{k}-u\right)\right\|^{2} \\
= & \beta_{k}\left\|\left[\gamma_{k} y_{k}+\left(1-\gamma_{k}\right) x_{k}\right]-u\right\|^{2}+\left(1-\beta_{k}\right)\left\|S^{k} y_{k}-u\right\|^{2} \\
\leqslant & \beta_{k}\left[\gamma_{k}\left\|y_{k}-u\right\|^{2}+\left(1-\gamma_{k}\right)\left\|x_{k}-u\right\|^{2}\right]+\left(1-\beta_{k}\right)\left\|S^{k} y_{k}-u\right\|^{2} \\
\leqslant & \beta_{k} \gamma_{k}\left\|y_{k}-u\right\|^{2}+\beta_{k}\left(1-\gamma_{k}\right)\left\|x_{k}-u\right\|^{2}+\left(1-\beta_{k}\right) s_{k}^{2}\left\|y_{k}-u\right\|^{2} \\
\leqslant & \beta_{k} \gamma_{k}\left[\left\|x_{k}-u\right\|^{2}+\left(1-\alpha_{k}\right)\left(t_{k}^{2}-1\right)\left\|x_{k}-u\right\|^{2}\right]+\beta_{k}\left(1-\gamma_{k}\right)\left\|x_{k}-u\right\|^{2} \\
& +\left(1-\beta_{k}\right) s_{k}^{2}\left[\left\|x_{k}-u\right\|^{2}+\left(1-\alpha_{k}\right)\left(t_{k}^{2}-1\right)\left\|x_{k}-u\right\|^{2}\right] \\
\leqslant & \beta_{k}\left\|x_{k}-u\right\|^{2}+\left(1-\alpha_{k}\right)\left(t_{k}^{2}-1\right)\left\|x_{k}-u\right\|^{2}+\left(1-\beta_{k}\right) s_{k}^{2}\left\|x_{k}-u\right\|^{2} \\
= & \left\|x_{k}-u\right\|^{2}-\left(1-\beta_{k}\right)\left\|x_{k}-u\right\|^{2}+\left(1-\alpha_{k}\right)\left(t_{k}^{2}-1\right)\left\|x_{k}-u\right\|^{2}+\left(1-\beta_{k}\right) s_{k}^{2}\left\|x_{k}-u\right\|^{2} \\
& +\left(1-\beta_{k}\right) s_{k}^{2}\left(1-\alpha_{k}\right)\left(t_{k}^{2}-1\right)\left\|x_{k}-u\right\|^{2} \\
= & \left\|x_{k}-u\right\|^{2}+\left(1-\beta_{k}\right)\left(s_{k}^{2}-1\right)\left\|x_{k}-u\right\|^{2}+\left(1-\alpha_{k}\right)\left(t_{k}^{2}-1\right)\left\|x_{k}-u\right\|^{2} \\
& +\left(1-\beta_{k}\right) s_{k}^{2}\left(1-\alpha_{k}\right)\left(t_{k}^{2}-1\right)\left\|x_{k}-u\right\|^{2} .
\end{aligned}
$$

From (3.1) and (3.2), we obtain that

$$
\left\|z_{k}-u\right\|^{2}+\left\|y_{k}-u\right\|^{2} \leqslant\left\|x_{k}-u\right\|^{2}+\left(1-\beta_{k}\right)\left(s_{k}^{2}-1\right)\left\|x_{k}-u\right\|^{2}+\left(1-\alpha_{k}\right)\left(t_{k}^{2}-1\right)\left\|x_{k}-u\right\|^{2}
$$




$$
\begin{aligned}
& \quad+\left(1-\beta_{k}\right) s_{k}^{2}\left(1-\alpha_{k}\right)\left(t_{k}^{2}-1\right)\left\|x_{k}-u\right\|^{2}+\left\|x_{k}-u\right\|^{2} \\
& \quad+\left(1-\alpha_{k}\right)\left(t_{k}^{2}-1\right)\left\|x_{k}-u\right\|^{2} \\
& \leqslant \\
& \quad 2\left\|x_{k}-u\right\|^{2}+\left[\left(1-\beta_{k}\right)\left(s_{k}^{2}-1\right)+2\left(1-\alpha_{k}\right)\left(t_{k}^{2}-1\right)\right. \\
& \left.\quad+\left(1-\beta_{k}\right) s_{k}^{2}\left(1-\alpha_{k}\right)\right](\operatorname{diam} C)^{2} \\
& =2\left\|x_{k}-u\right\|^{2}+\theta_{k},
\end{aligned}
$$

where $\theta_{k}=\left[\left(1-\beta_{k}\right)\left(s_{k}^{2}-1\right)+2\left(1-\alpha_{k}\right)\left(t_{k}^{2}-1\right)+\left(1-\beta_{k}\right) s_{k}^{2}\left(1-\alpha_{k}\right)\right](\text { diamC })^{2} \rightarrow 0$ as $n \rightarrow \infty$. It follows that $u \in C_{k+1}$ and then $F(T) \cap F(S) \subseteq C_{n}$ for all $n \in \mathbb{N}$. Next, we show that $C_{n}$ is closed and convex for all $n \in \mathbb{N}$. It is obvious that $C_{1}=C$ is closed and convex. Suppose that $C_{k}$ is closed and convex for each $k \in \mathbb{N}$. Let $\left\{z_{m}\right\}_{m=1}^{\infty} \subseteq C_{k+1} \subseteq C_{k}$ with $z_{m} \rightarrow z$ as $m \rightarrow \infty$. Since $C_{k}$ is closed and $z_{m} \in C_{k+1}$, we have $z_{m} \in C_{k}$ and $\left\|z_{k}-z_{m}\right\|^{2}+\left\|y_{k}-z_{m}\right\|^{2} \leqslant 2\left\|z_{m}-x_{k}\right\|^{2}+\theta_{k}$. From Lemma 2.1, we have

$$
\begin{aligned}
\left\|z_{k}-z\right\|^{2}+\left\|y_{k}-z\right\|^{2}= & \left\|z_{k}-z_{m}+z_{m}-z\right\|^{2}+\left\|y_{k}-z_{m}+z_{m}-z\right\|^{2} \\
\leqslant & {\left[\left\|z_{k}-z_{m}\right\|^{2}+\left\|z_{m}-z\right\|^{2}+2\left\langle z_{k}-z_{m}, z_{m}-z\right\rangle\right] } \\
& +\left[\left\|y_{k}-z_{m}\right\|^{2}+\left\|z_{m}-z\right\|^{2}+2\left\langle y_{k}-z_{m}, z_{m}-z\right\rangle\right] \\
= & \left\|z_{k}-z_{m}\right\|^{2}+\left\|y_{k}-z_{m}\right\|^{2}+2\left\|z_{m}-z\right\|^{2} \\
& +2\left(\left\|z_{k}-z_{m}\right\|\left\|z_{m}-z\right\|+\left\|y_{k}-z_{m}\right\|\left\|z_{m}-z\right\|\right) \\
\leqslant & 2\left\|x_{k}-z_{m}\right\|^{2}+\theta_{k} \\
& +2\left(\left\|z_{m}-z\right\|^{2}+\left\|z_{k}-z_{m}\right\|\left\|z_{m}-z\right\|+\left\|y_{k}-z_{m}\right\|\left\|z_{m}-z\right\|\right) .
\end{aligned}
$$

Taking $\mathrm{m} \rightarrow \infty$, it follows that

$$
\left\|z_{k}-z\right\|^{2}+\left\|y_{k}-z\right\|^{2} \leqslant 2\left\|x_{k}-z\right\|^{2}+\theta_{k} .
$$

Then $z \in C_{k+1}$ and hence $C_{k+1}$ is closed. Let $x, y \in C_{k+1} \subseteq C_{k}$ with $z=\alpha x+(1-\alpha) y$ where $\alpha \in[0,1]$. Since $C_{k}$ is convex, $z \in C_{k}$. Thus, we have

$$
\left\|z_{k}-x\right\|^{2}+\left\|y_{k}-x\right\|^{2} \leqslant 2\left\|x_{k}-x\right\|^{2}+\theta_{k},
$$

and

$$
\left\|z_{k}-y\right\|^{2}+\left\|y_{k}-y\right\|^{2} \leqslant 2\left\|x_{k}-y\right\|^{2}+\theta_{k} .
$$

Hence

$$
\begin{aligned}
\left\|z_{k}-z\right\|^{2}+\left\|y_{k}-z\right\|^{2}= & \left\|z_{k}-(\alpha x+(1-\alpha) y)\right\|^{2}+\left\|y_{k}-(\alpha x+(1-\alpha) y)\right\|^{2} \\
= & \left\|\alpha\left(z_{k}-x\right)+(1-\alpha)\left(z_{k}-y\right)\right\|^{2}+\left\|\alpha\left(y_{k}-x\right)+(1-\alpha)\left(y_{k}-y\right)\right\|^{2} \\
= & \alpha\left\|z_{k}-x\right\|^{2}+(1-\alpha)\left\|z_{k}-y\right\|^{2}-\alpha(1-\alpha)\|x-y\|^{2} \\
& +\alpha\left\|y_{k}-x\right\|^{2}+(1-\alpha)\left\|y_{k}-y\right\|^{2}-\alpha(1-\alpha)\|x-y\|^{2} \\
= & \alpha\left(\left\|z_{k}-x\right\|^{2}+\left\|y_{k}-x\right\|^{2}\right)+(1-\alpha)\left(\left\|z_{k}-y\right\|^{2}+\|y k-y\|^{2}\right) \\
& -2 \alpha(1-\alpha)\|x-y\|^{2} \\
\leqslant & \alpha\left(2\left\|x_{k}-x\right\|^{2}+\theta_{k}\right)+(1-\alpha)\left(2\left\|x_{k}-y\right\|^{2}+\theta_{k}\right)-2 \alpha(1-\alpha)\|x-y\|^{2} \\
= & 2\left[\alpha\left\|x_{k}-x\right\|^{2}+(1-\alpha)\left\|x_{k}-y\right\|^{2}-\alpha(1-\alpha)\|x-y\|^{2}\right] \\
& +\alpha \theta_{k}+(1-\alpha) \theta_{k} \\
= & 2\left\|\alpha\left(x_{k}-x\right)+(1-\alpha)\left(x_{k}-y\right)\right\|^{2}+\theta_{k} \\
= & 2\left\|x_{k}-(\alpha x+(1-\alpha) y)\right\|^{2} \\
= & 2\left\|x_{k}-z\right\|^{2}+\theta_{k} .
\end{aligned}
$$


It follows that $z \in C_{k+1}$ and hence $C_{k+1}$ is convex. Therefore, $C_{n}$ is closed and convex for all $n \in \mathbb{N}$. This implies that $\left\{x_{n}\right\}$ is well-defined. Since $x_{n}=P_{C_{n}} x_{0}$, it follows that

$$
\left\langle x_{0}-x_{n}, x_{n}-y\right\rangle \geqslant 0,
$$

for all $y \in F(T) \cap F(S)$ and $n \in \mathbb{N}$. So $u \in F(T) \cap F(S)$, we have

$$
\begin{aligned}
0 & \leqslant\left\langle x_{0}-x_{n}, x_{n}-u\right\rangle=-\left\langle x_{n}-x_{0}, x_{n}-x_{0}\right\rangle+\left\langle x_{0}-x_{n}, x_{0}-u\right\rangle \\
& \leqslant-\left\|x_{n}-x_{0}\right\|^{2}+\left\|x_{0}-x_{n}\right\|\left\|x_{0}-u\right\| .
\end{aligned}
$$

This implies that

$$
\left\|x_{0}-x_{n}\right\|^{2} \leqslant\left\|x_{0}-x_{n}\right\|\left\|x_{0}-u\right\|,
$$

and hence

$$
\left\|x_{0}-x_{n}\right\| \leqslant\left\|x_{0}-u\right\|,
$$

for all $u \in F(T) \cap F(S)$ and $n \in \mathbb{N}$. From $x_{n}=P_{C_{n}} x_{0}$ and $x_{n+1}=P_{C_{n+1}} x_{0} \in C_{n+1} \subseteq C_{n}$, we obtain that

$$
\left\langle x_{0}-x_{n}, x_{n}-x_{n+1}\right\rangle \geqslant 0,
$$

for all $n \in \mathbb{N}$. So, for all $x_{n+1} \in C_{n+1}$, for $n \in \mathbb{N}$, we have

$$
\begin{aligned}
0 & \leqslant\left\langle x_{0}-x_{n}, x_{n}-x_{n+1}\right\rangle=-\left\langle x_{n}-x_{0}, x_{n}-x_{0}\right\rangle+\left\langle x_{0}-x_{n}, x_{0}-x_{n+1}\right\rangle \\
& \leqslant-\left\|x_{n}-x_{0}\right\|^{2}+\left\|x_{0}-x_{n}\right\|\left\|x_{0}-x_{n+1}\right\| .
\end{aligned}
$$

This implies that

$$
\left\|x_{0}-x_{n}\right\|^{2} \leqslant\left\|x_{0}-x_{n}\right\|\left\|x_{0}-x_{n+1}\right\|
$$

and hence

$$
\left\|x_{0}-x_{n}\right\| \leqslant\left\|x_{0}-x_{n+1}\right\|,
$$

for all $n \in \mathbb{N}$. Since $\left\{\left\|x_{0}-x_{n}\right\|\right\}$ is bounded, $\lim _{n \rightarrow \infty}\left\|x_{n}-x_{0}\right\|$ exists. Next, we claim that

$$
\lim _{n \rightarrow \infty}\left\|x_{n}-x_{n+1}\right\|=0
$$

From (3.3), we have

$$
\begin{aligned}
\left\|x_{n}-x_{n+1}\right\|^{2} & =\left\|\left(x_{n}-x_{0}\right)+\left(x_{0}-x_{n+1}\right)\right\|^{2} \\
& =\left\|x_{n}-x_{0}\right\|^{2}+2\left\langle x_{n}-x_{0}, x_{0}-x_{n+1}\right\rangle+\left\|x_{0}-x_{n+1}\right\|^{2} \\
& =\left\|x_{n}-x_{0}\right\|^{2}-2\left\langle x_{0}-x_{n}, x_{0}-x_{n}\right\rangle-2\left\langle x_{0}-x_{n}, x_{n}-x_{n+1}\right\rangle+\left\|x_{0}-x_{n+1}\right\|^{2} \\
& \leqslant\left\|x_{n}-x_{0}\right\|^{2}-2\left\|x_{n}-x_{0}\right\|^{2}+\left\|x_{0}-x_{n+1}\right\|^{2} \\
& =-\left\|x_{n}-x_{0}\right\|^{2}+\left\|x_{0}-x_{n+1}\right\|^{2} .
\end{aligned}
$$

Since, $\lim _{n \rightarrow \infty}\left\|x_{n}-x_{0}\right\|$ exists, we have $\lim _{n \rightarrow \infty}\left\|x_{n}-x_{n+1}\right\|=0$. Next, we now claim that

$$
\lim _{n \rightarrow \infty}\left\|T x_{n}-x_{n}\right\|=0=\lim _{n \rightarrow \infty}\left\|S x_{n}-x_{n}\right\| .
$$

Since $x_{n+1} \in C_{n}$, we have

$$
\left\|z_{n}-x_{n+1}\right\|^{2}+\left\|y_{n}-x_{n+1}\right\|^{2} \leqslant 2\left\|x_{n}-x_{n+1}\right\|^{2}+\theta_{n}
$$

From $\lim _{n \rightarrow \infty}\left\|x_{n}-x_{n+1}\right\|=0$ and $\theta_{n} \rightarrow 0$ as $n \rightarrow \infty$, it follows that

$$
\lim _{n \rightarrow \infty}\left\|z_{n}-x_{n+1}\right\|=0=\lim _{n \rightarrow \infty}\left\|y_{n}-x_{n+1}\right\|,
$$


which yields

and then we have

$$
\left\|z_{n}-x_{n}\right\| \leqslant\left\|z_{n}-x_{n+1}\right\|+\left\|x_{n+1}-x_{n}\right\| \rightarrow 0, \quad \text { as } n \rightarrow \infty,
$$

$$
\left\|y_{n}-x_{n}\right\| \leqslant\left\|y_{n}-x_{n+1}\right\|+\left\|x_{n+1}-x_{n}\right\| \rightarrow 0, \quad \text { as } n \rightarrow \infty .
$$

By definition of $y_{n}$, we have $y_{n}-x_{n}=\left(1-\alpha_{n}\right)\left(T^{n} x_{n}-x_{n}\right)$, we obtain

$$
\left\|T^{n} x_{n}-x_{n}\right\|=\frac{1}{1-\alpha_{n}}\left\|y_{n}-x_{n}\right\|
$$

Since $\alpha_{\mathrm{n}} \leqslant 1-\delta$, then we have

$$
\left\|T^{n} x_{n}-x_{n}\right\| \rightarrow 0,
$$

as $n \rightarrow \infty$. From $z_{n}=\beta_{n}\left[\gamma_{n} y_{n}+\left(1-\gamma_{n}\right) x_{n}\right]+\left(1-\beta_{n}\right) S^{n} y_{n}$, we have

$$
\begin{aligned}
\left\|S^{n} y_{n}-z_{n}\right\| & =\frac{\beta_{n}}{1-\beta_{n}}\left\|\gamma_{n}\left(y_{n}-z_{n}\right)+\left(1-\gamma_{n}\right)\left(x_{n}-z_{n}\right)\right\| \\
& \leqslant \frac{1}{1-\beta_{n}}\left(\gamma_{n}\left\|y_{n}-z_{n}\right\|+\left(1-\gamma_{n}\right)\left\|x_{n}-z_{n}\right\|\right),
\end{aligned}
$$

which yields

$$
\left\|S^{n} y_{n}-z_{n}\right\| \rightarrow 0, \quad \text { as } n \rightarrow \infty
$$

and so

$$
\begin{aligned}
\left\|T x_{n}-x_{n}\right\| & \leqslant\left\|T x_{n}-T^{n+1} x_{n}\right\|+\left\|T^{n+1} x_{n}-T^{n+1} x_{n+1}\right\|+\left\|T^{n+1} x_{n+1}-x_{n+1}\right\|+\left\|x_{n+1}-x_{n}\right\| \\
& \leqslant t_{\infty}\left\|x_{n}-T^{n} x_{n}\right\|+\left\|T^{n+1} x_{n+1}-x_{n+1}\right\|+\left(1+t_{\infty}\right)\left\|x_{n}-x_{n+1}\right\| \rightarrow 0, \quad \text { as } n \rightarrow \infty
\end{aligned}
$$

Similarly, we have

$$
\left\|S x_{n}-x_{n}\right\| \rightarrow 0, \quad \text { as } n \rightarrow \infty .
$$

By Lemma 2.4, and boundedness of $\left\{x_{n}\right\}$, we have $\emptyset \neq \omega_{w}\left(x_{n}\right) \subset F(T) \cap F(S)$. Since

$$
z_{0}=\mathrm{P}_{\mathrm{F}(\mathrm{T}) \cap \mathrm{F}(\mathrm{S})} \mathrm{x}_{0}, z_{0} \in \mathrm{F}(\mathrm{T}) \cap \mathrm{F}(\mathrm{S}) \subset \mathrm{C},
$$

and Lemma 2.5 guarantees the strong convergence of $\left\{x_{n}\right\}$ to $P_{F(T) \cap F(S)} x_{0}$. This completes the proof.

\subsection{Strong convergence theorem of asymptotically nonexpansive semigroups}

First, we study some examples for relationship between a nonexpansive semigroup and an asymptotically nonexpansive semigroup for motivation of this work.

Example 3.2. Let $\mathrm{H}_{1}=\mathrm{H}_{2}=\mathbb{R}$ and let $\mathcal{T}:=\{\mathrm{T}(\mathrm{s}): 0 \leqslant s<\infty\}$, where

$$
\mathrm{T}(\mathrm{s}) \mathrm{x}=\frac{1}{1+2 \mathrm{~s}} x, \quad \forall x \in \mathbb{R} .
$$

We see that for any $x, y \in \mathbb{R}$

$$
\|\mathrm{T}(\mathrm{s}) \mathrm{x}-\mathrm{T}(\mathrm{s}) \mathrm{y}\|=\left\|\left(\frac{1}{1+2 \mathrm{~s}}\right) x-\left(\frac{1}{1+2 \mathrm{~s}}\right) y\right\|=\left(\frac{1}{1+2 \mathrm{~s}}\right)\|x-y\|,
$$

then we have $\mathcal{T}$ is nonexpansive semigroup. If $L_{s}=1$ we have $\lim \sup _{s \rightarrow \infty} L_{s}=1$, then $\mathcal{T}$ is asymptotically nonexpansive semigroup.

Example 3.3. Let $\mathrm{H}_{1}=\mathrm{H}_{2}=\mathbb{R}$ and let $\mathcal{T}:=\{\mathrm{T}(\mathrm{s}): 0 \leqslant \mathrm{~s}<\infty\}$, where

$$
\mathrm{T}(\mathrm{s}) x=\frac{2+2 \mathrm{~s}}{1+2 \mathrm{~s}} x, \quad \forall x \in \mathbb{R} .
$$

We see that for any $x, y \in \mathbb{R}$

$$
\|\mathrm{T}(\mathrm{s}) x-\mathrm{T}(\mathrm{s}) \mathrm{y}\|=\left\|\left(\frac{2+2 \mathrm{~s}}{1+2 \mathrm{~s}}\right) x-\left(\frac{2+2 \mathrm{~s}}{1+2 \mathrm{~s}}\right) y\right\|=\left(\frac{2+2 \mathrm{~s}}{1+2 \mathrm{~s}}\right)\|x-y\|,
$$

put $L_{s}=\left(\frac{2+2 s}{1+2 s}\right)$ we have $\lim \sup _{s \rightarrow \infty} L_{s}=\limsup _{s \rightarrow \infty}\left(\frac{2+2 s}{1+2 s}\right)=1$, then $\mathcal{T}$ is asymptotically nonexpansive 
semigroup. If we let $s=1$ we have $\frac{2+2 s}{1+2 s}=\frac{4}{3} \nless 1$, then $\mathcal{T}$ is not necessarily nonexpansive semigroup.

From above example we see that a mapping $\mathcal{T}$ is a nonexpansive semigroup then $\mathcal{T}$ is asymptotically nonexpansive semigroup. But $\mathcal{T}$ is an asymptotically nonexpansive semigroup is not nonexpansive semigroup.

In 2008, Takahashi et al. [11] proved the strong convergence theorems by the hybrid method for nonexpansive semigroup in Hilbert space.

Theorem 3.4 ([11]). Let $\mathrm{H}$ be a Hilbert space and $\mathrm{C}$ be a nonempty closed convex subset of $\mathrm{H}$. Let

$$
\mathcal{T}=\{\mathrm{T}(\mathrm{s}): 0 \leqslant s<\infty\},
$$

be a one-parameter nonexpansive mapping semigroup on $\mathrm{C}$ such that $\mathrm{F}(\mathcal{T}) \neq \emptyset$ and let $\mathrm{x}_{0} \in \mathrm{H}$. For $\mathrm{C}_{1}=\mathrm{C}$ and $\mathrm{u}_{1}=\mathrm{P}_{\mathrm{C}_{1}} \mathrm{x}_{0}$, define a sequence $\left\{\mathrm{u}_{\mathrm{n}}\right\}$ of $\mathrm{C}$ as follows:

$$
\left\{\begin{array}{l}
y_{n}=\alpha_{n} u_{n}+\left(1-\alpha_{n}\right) \frac{1}{\lambda_{n}} \int_{0}^{\lambda_{n}} T(s) u_{n} d s, \\
C_{n+1}=\left\{z \in C_{n}:\left\|y_{n}-z\right\| \leqslant\left\|u_{n}-z\right\|\right\}, \\
u_{n+1}=P_{C_{n+1}} x_{0}, n \in \mathbb{N},
\end{array}\right.
$$

where $0 \leqslant \alpha_{n} \leqslant a<1,0<\lambda_{n}<\infty$ for all $n \in \mathbb{N}$ and $\lambda_{n} \rightarrow \infty$. Then $\left\{u_{n}\right\}$ converges strongly to $z_{0}=P_{F(\mathcal{T})} x_{0}$.

In the same year, Inchan and Plubtieng [3] modified Ishikawa iteration process for two asymptotically nonexpansive semigroups for $\mathrm{C}$ is a nonempty closed convex subset of a Hilbert space $\mathrm{H}$,

$$
\mathcal{T}=\{\mathrm{T}(\mathrm{t}): 0 \leqslant \mathrm{t}<\infty\},
$$

and

$$
\mathcal{S}=\{\mathrm{S}(\mathrm{t}): 0 \leqslant \mathrm{t}<\infty\},
$$

be two asymptotically nonexpansive semigroups on $C$ such that $\mathcal{F}=F(\mathcal{T}) \cap F(\mathcal{S}) \neq \emptyset$ and let $x_{0} \in C$. For $\mathrm{C}_{1}=\mathrm{C}$ and $\mathrm{x}_{1}=\mathrm{P}_{\mathrm{C}_{1}}$, define $\left\{\mathrm{x}_{\mathrm{n}}\right\}$ as follows:

$$
\left\{\begin{array}{l}
y_{n}=\alpha_{n} x_{n}+\left(1-\alpha_{n}\right) \frac{1}{t_{n}} \int_{0}^{t_{n}} T(t) z_{n} d t \\
z_{n}=\beta_{n} x_{n}+\left(1-\beta_{n}\right) \frac{1}{t_{n}} \int_{0}^{t_{n}} S(t) x_{n} d t \\
C_{n+1}=\left\{z \in C_{n}:\left\|y_{n}-z\right\|^{2} \leqslant\left\|x_{n}-z\right\|^{2}+\widetilde{\theta_{n}}\right\} \\
x_{n+1}=P_{C_{n+1}} x_{0}, n \in \mathbb{N},
\end{array}\right.
$$

where

$$
\widetilde{\theta_{n}}=\left(1-\alpha_{n}\right)\left[\left(\widetilde{t}_{n}^{2}-1\right)+\left(1-\beta_{n}\right) \widetilde{t}_{n}^{2}\left(\widetilde{s}_{n}^{2}-1\right)\right](\operatorname{diamC})^{2} \rightarrow 0,
$$

(here $\widetilde{t}_{n}=\frac{1}{t_{n}} \int_{0}^{t_{n}} L_{t}^{\top} d t$ and $\widetilde{s}_{n} \frac{1}{s_{n}} \int_{0}^{s_{n}} L_{t}^{S} d t$ and $0 \leqslant \alpha_{n} \leqslant a<1$ and $0<b \leqslant \beta_{n} \leqslant c<1$ for all $n \in \mathbb{N}$ and $\widetilde{\mathrm{t}}_{\mathrm{n}} \rightarrow \infty, \widetilde{\mathrm{s}}_{\mathrm{n}} \rightarrow \infty$ ).

As the second part of this work, we extend the results of Dang, et al. [1] for $\mathcal{T}$ is an asymptotically nonexpansive semigroup, then we consider

$$
\left\{\begin{array}{l}
x_{0} \in C=C_{1}, x_{1}=P_{C_{1}} x_{0}, \\
y_{n}=\alpha_{n} x_{n}+\left(1-\alpha_{n}\right) \frac{1}{t_{n}} \int_{0}^{t_{n}} T(t) z_{n} d t \\
z_{n}=\beta_{n}\left[\gamma_{n} y_{n}+\left(1-\gamma_{n}\right) x_{n}\right]+\left(1-\beta_{n}\right) \frac{1}{s_{n}} \int_{0}^{s_{n}} S(t) x_{n} d t \\
C_{n+1}=\left\{z \in C_{n}:\left\|z_{n}-z\right\|^{2}+\left\|y_{n}-z\right\|^{2} \leqslant 2\left\|x_{n}-z\right\|^{2}+\widetilde{\theta_{n}}\right\}, \\
x_{n+1}=P_{C_{n+1}} x_{0}, n \geqslant 0,
\end{array}\right.
$$

where $\alpha_{n}, \beta_{n}, \gamma_{n} \in[0,1]$ and

$$
\widetilde{\theta_{n}}=\left[\left(1-\beta_{n}\right)\left(\widetilde{s}_{n}^{2}-1\right)+\left(1-\alpha_{n}\right)\left(\widetilde{t}_{n}^{2}-1\right)+\left(1-\beta_{n}\right) \widetilde{s}_{n}^{2}\left(1-\alpha_{n}\right)\left(\widetilde{t}_{n}^{2}-1\right)\right](\operatorname{diamC})^{2} \rightarrow 0,
$$

as $n \rightarrow \infty$ (here $\widetilde{t}_{n}=\frac{1}{t_{n}} \int_{0}^{t_{n}} L_{t}^{\top} d t \rightarrow 1$ and $\widetilde{s}_{n} \frac{1}{s_{n}} \int_{0}^{s_{n}} L_{t}^{S} d t \rightarrow 1$ as $n \rightarrow \infty$ ). 
Theorem 3.5. Let $\mathrm{H}$ be a Hilbert space and let $\mathrm{C}$ be a nonempty closed bounded subset of $\mathrm{H}$. Let

$$
\mathfrak{T}=\{\mathbf{T}(\mathrm{t}): 0 \leqslant \mathrm{t}<\infty\},
$$

and

$$
\mathfrak{S}=\{\mathrm{S}(\mathrm{t}): 0 \leqslant \mathrm{t}<\infty\},
$$

be two asymptotically nonexpansive semigroups on $\mathrm{C}$ such that $\mathfrak{F}=\mathrm{F}(\mathfrak{T}) \cap \mathrm{F}(\mathfrak{S}) \neq \emptyset$ and let $\mathrm{x}_{0} \in \mathrm{C}$. Let $C_{1}=C, x_{1}=P_{C_{1}} x_{0}$ and $\left\{x_{n}\right\}$ be a sequence generated by (3.4) with satisfies $\alpha_{n}, \beta_{n}, \gamma_{n} \in[0,1], 0 \leqslant \alpha_{n} \leqslant a<1$ and $0<\mathrm{b} \leqslant \beta_{\mathrm{n}} \leqslant \mathrm{c}<1$ for all $\mathrm{n} \in \mathbb{N} \cup\{0\}$ and $\mathrm{t}_{\mathrm{n}} \rightarrow \infty, \mathrm{s}_{\mathrm{n}} \rightarrow \infty$. Then $\left\{\mathrm{x}_{\mathrm{n}}\right\}$ converges strongly to $z_{0}=\mathrm{P}_{\mathfrak{F}} \mathrm{x}_{0}$.

Proof. First observe that $\mathfrak{F} \subseteq C_{n}$ for all $n \in \mathbb{N}$. For $\mathfrak{F} \subset \mathrm{C}=\mathrm{C}_{1}$ suppose that $\mathfrak{F} \subset \mathrm{C}_{\mathrm{k}}$ for each $k \in \mathbb{N}$. Let $\mathrm{u} \in \mathfrak{F} \subset \mathrm{C}_{\mathrm{k}}$. Then we have

$$
\begin{aligned}
\left\|y_{k}-u\right\|^{2} & =\left\|\alpha_{k} x_{k}+\left(1-\alpha_{k}\right) \frac{1}{t_{k}} \int_{0}^{t_{k}} T(t) x_{k} d t-u\right\|^{2} \\
& =\left\|\alpha_{k}\left(x_{k}-u\right)+\left(1-\alpha_{k}\right)\left(\frac{1}{t_{k}} \int_{0}^{t_{k}} T(t) x_{k} d t-u\right)\right\|^{2} \\
& \leqslant \alpha_{k}\left\|x_{k}-u\right\|^{2}+\left(1-\alpha_{k}\right)\left\|\frac{1}{t_{k}} \int_{0}^{t_{k}} T(t) x_{k} d t-u\right\|^{2} \\
& \leqslant \alpha_{k}\left\|x_{k}-u\right\|^{2}+\left(1-\alpha_{k}\right)\left(\frac{1}{t_{k}} \int_{0}^{t_{k}}\left\|T(t) x_{k}-u\right\| d t\right)^{2} \\
& \leqslant \alpha_{k}\left\|x_{k}-u\right\|^{2}+\left(1-\alpha_{k}\right)\left(\frac{1}{t_{k}} \int_{0}^{t_{k}} L_{t}^{\top}\left\|x_{k}-u\right\| d t\right)^{2} \\
& \leqslant \alpha_{k}\left\|x_{k}-u\right\|^{2}+\left(1-\alpha_{k}\right)\left(\frac{1}{t_{k}} \int_{0}^{t_{k}} L_{t}^{\top} d t\right)^{2}\left\|x_{k}-u\right\|^{2} \\
& =\left\|x_{k}-u\right\|^{2}+\left(1-\alpha_{k}\right)\left({\widetilde{t_{k}}}^{2}-1\right)\left\|x_{k}-u\right\|^{2} .
\end{aligned}
$$

By Lemma 2.1 again, we have

$$
\begin{aligned}
\left\|z_{k}-u\right\|^{2}= & \left\|\beta_{k}\left[\gamma_{k} y_{k}+\left(1-\gamma_{k}\right) x_{k}\right]+\left(1-\beta_{k}\right) \frac{1}{s_{k}} \int_{0}^{s_{k}} S(t) y_{k} d t-u\right\|^{2} \\
= & \left\|\beta_{k}\left(\left[\gamma_{k} y_{k}+\left(1-\gamma_{k}\right) x_{k}\right]-u\right)+\left(1-\beta_{k}\right)\left(\frac{1}{s_{k}} \int_{0}^{s_{k}} S(t) y_{k} d t-u\right)\right\|^{2} \\
\leqslant & \beta_{k}\left\|\left[\gamma_{k} y_{k}+\left(1-\gamma_{k}\right) x_{k}\right]-u\right\|^{2}+\left(1-\beta_{k}\right)\left\|\frac{1}{s_{k}} \int_{0}^{s_{k}} S(t) y_{k} d t-u\right\|^{2} \\
\leqslant & \beta_{k}\left\|\gamma_{k}\left(y_{k}-u\right)+\left(1-\gamma_{k}\right)\left(x_{k}-u\right)\right\|^{2}+\left(1-\beta_{k}\right)\left(\frac{1}{s_{k}} \int_{0}^{s_{k}}\left\|S(t) y_{k}-u\right\| d t\right)^{2} \\
\leqslant & \beta_{k} \gamma_{k}\left\|y_{k}-u\right\|^{2}+\beta_{k}\left(1-\gamma_{k}\right)\left\|x_{k}-u\right\|^{2}+\left(1-\beta_{k}\right)\left(\frac{1}{s_{k}} \int_{0}^{s_{k}} L_{t}^{S}\left\|y_{k}-u\right\| d t\right)^{2} \\
\leqslant & \beta_{k} \gamma_{k}\left\|y_{k}-u\right\|^{2}+\beta_{k}\left(1-\gamma_{k}\right)\left\|x_{k}-u\right\|^{2}+\left(1-\beta_{k}\right)\left(\frac{1}{s_{k}} \int_{0}^{s_{k}} L_{t}^{S} d t\right)^{2}\left\|y y_{k}-u\right\|^{2} \\
\leqslant & \beta_{k} \gamma_{k}\left[\left\|x_{k}-u\right\|^{2}+\left(1-\alpha_{k}\right)\left(\widetilde{t_{k}}-1\right)\left\|x_{k}-u\right\|^{2}\right]+\beta_{k}\left(1-\gamma_{k}\right)\left\|x_{k}-u\right\|^{2} \\
& +\left(1-\beta_{k}\right) \widetilde{s_{k}}{ }^{2}\left[\left\|x_{k}-u\right\|^{2}+\left(1-\alpha_{k}\right)\left(\widetilde{t_{k}}-1\right)\left\|x_{k}-u\right\|^{2}\right] \\
\leqslant & \beta_{k}\left\|x_{k}-u\right\|^{2}+\left(1-\alpha_{k}\right)\left(\widetilde{t_{k}}-1\right)\left\|x_{k}-u\right\|^{2}+\left(1-\beta_{k}\right) \widetilde{s_{k}}\left\|x_{k}-u\right\|^{2} \\
& +\left(1-\beta_{k}\right) \widetilde{s_{k}}{ }^{2}\left(1-\alpha_{k}\right)\left(\widetilde{t_{k}}-1\right)\left\|x_{k}-u\right\|^{2} \\
= & \left\|x_{k}-u\right\|^{2}+\left(1-\beta_{k}\right)\left(\widetilde{s_{k}}{ }^{2}-1\right)\left\|x_{k}-u\right\|^{2}+\left(1-\alpha_{k}\right)\left(\widetilde{t_{k}}{ }^{2}-1\right)\left\|x_{k}-u\right\|^{2} \\
& +\left(1-\beta_{k}\right){\widetilde{s_{k}}}^{2}\left(1-\alpha_{k}\right)\left(\widetilde{t_{k}}-1\right)\left\|x_{k}-u\right\|^{2} .
\end{aligned}
$$


From (3.5) and (3.6), we obtain that

$$
\begin{aligned}
\left\|z_{k}-u\right\|^{2}+\left\|y_{k}-u\right\|^{2} \leqslant & \left\|x_{k}-u\right\|^{2}+\left(1-\beta_{k}\right)\left(\widetilde{s_{k}}{ }^{2}-1\right)\left\|x_{k}-u\right\|^{2}+\left(1-\alpha_{k}\right)\left(\widetilde{t_{k}}-1\right)\left\|x_{k}-u\right\|^{2} \\
& +\left(1-\beta_{k}\right){\widetilde{s_{k}}}^{2}\left(1-\alpha_{k}\right)\left(\widetilde{t_{k}}-1\right)\left\|x_{k}-u\right\|^{2}+\left\|x_{k}-u\right\|^{2} \\
& +\left(1-\alpha_{k}\right)\left(\widetilde{t_{k}}-1\right)\left\|x_{k}-u\right\|^{2}+\left(1-\beta_{k}\right) s_{k}^{2}\left(1-\alpha_{k}\right)\left(t_{k}^{2}-1\right)\left\|x_{k}-u\right\|^{2} \\
& +\left\|x_{k}-u\right\|^{2}+\left(1-\alpha_{k}\right)\left(\tilde{t}_{k}^{2}-1\right)\left\|x_{k}-u\right\|^{2} \\
\leqslant & 2\left\|x_{k}-u\right\|^{2}+\left[\left(1-\beta_{k}\right)\left(\widetilde{s_{k}}-1\right)+2\left(1-\alpha_{k}\right)\left(\widetilde{t_{k}}-1\right)\right. \\
& \left.+\left(1-\beta_{k}\right){\widetilde{s_{k}}}^{2}\left(1-\alpha_{k}\right)\left(\widetilde{t_{k}}{ }^{2}-1\right)\right](\operatorname{diamC})^{2} \\
= & 2\left\|x_{k}-u\right\|^{2}+\widetilde{\theta_{k}}
\end{aligned}
$$

where

$$
\widetilde{\theta_{k}}=\left[\left(1-\beta_{k}\right)\left(\widetilde{s}_{k}^{2}-1\right)+2\left(1-\alpha_{k}\right)\left(\widehat{t}_{k}^{2}-1\right)+\left(1-\beta_{k}\right) \widetilde{s}_{k}^{2}\left(1-\alpha_{k}\right)\left(\widetilde{t}_{k}^{2}-1\right)\right](\operatorname{diamC})^{2} \rightarrow 0,
$$

as $n \rightarrow \infty$ (here $\widetilde{t}_{k}=\frac{1}{t_{k}} \int_{0}^{t_{k}} L_{t}^{T} d t \rightarrow 1$ and $\widetilde{s}_{k} \frac{1}{s_{k}} \int_{0}^{s_{k}} L_{t}^{S} d t \rightarrow 1$ as $k \rightarrow \infty$ ). It follows that $u \in C_{k+1}$ and then $F(T) \cap F(S) \subseteq C_{n}$ for all $n \in \mathbb{N}$. Again, by using the same argument in the proof in Theorem 3.1, we can show that $C_{n}$ is closed and convex for all $n \in \mathbb{N}$. It is obvious that $C_{1}=C$ is closed and convex. Suppose that $C_{k}$ is closed and convex for each $k \in \mathbb{N}$. Let $\left\{z_{m}\right\}_{m=1}^{\infty} \subseteq C_{k+1} \subseteq C_{k}$ with $z_{m} \rightarrow z$ as $m \rightarrow \infty$. Since $C_{k}$ is closed and $z_{m} \in C_{k+1}$, we have $z \in C_{k}$ and $\left\|z_{k}-z_{m}\right\|^{2} \leqslant\left\|z_{m}-x_{k}\right\|^{2}+\widetilde{\theta}_{k}$. From Lemma 2.1, we have

$$
\begin{aligned}
\left\|z_{k}-z\right\|^{2}+\left\|y_{k}-z\right\|^{2}= & \left\|z_{k}-z_{m}+z_{m}-z\right\|^{2}+\left\|y_{k}-z_{m}+z_{m}-z\right\|^{2} \\
\leqslant & {\left[\left\|z_{k}-z_{m}\right\|^{2}+\left\|z_{m}-z\right\|^{2}+2\left\langle z_{k}-z_{m}, z_{m}-z\right\rangle\right] } \\
& +\left[\left\|y_{k}-z_{m}\right\|^{2}+\left\|z_{m}-z\right\|^{2}+2\left\langle y_{k}-z_{m}, z_{m}-z\right\rangle\right] \\
= & \left\|z_{k}-z_{m}\right\|^{2}+\left\|y_{k}-z_{m}\right\|^{2}+2\left\|z_{m}-z\right\|^{2} \\
& +2\left(\left\|z_{k}-z_{m}\right\|\left\|z_{m}-z\right\|+\left\|y_{k}-z_{m}\right\|\left\|z_{m}-z\right\|\right) \\
\leqslant & 2\left\|x_{k}-z_{m}\right\|^{2}+\widetilde{\theta}_{k}+2\left(\left\|z_{m}-z\right\|^{2}\right. \\
& \left.+\left\|z_{k}-z_{m}\right\|\left\|z_{m}-z\right\|+\left\|y_{k}-z_{m}\right\|\left\|z_{m}-z\right\|\right) .
\end{aligned}
$$

Taking $\mathrm{m} \rightarrow \infty$, it follows that

$$
\left\|z_{k}-z\right\|^{2}+\left\|y_{k}-z\right\|^{2} \leqslant 2\left\|x_{k}-z\right\|^{2}+\widetilde{\theta}_{k}
$$

Then $z \in C_{k+1}$ and hence $C_{k+1}$ is closed. Let $x, y \in C_{k+1} \subseteq C_{k}$ with $z=\alpha x+(1-\alpha) y$ where $\alpha \in[0,1]$. Since $C_{k}$ is convex, $z \in C_{k}$. Thus, we have

$$
\left\|z_{k}-x\right\|^{2}+\left\|y_{k}-x\right\|^{2} \leqslant 2\left\|x_{k}-x\right\|^{2}+\widetilde{\theta}_{k},
$$

and

$$
\left\|z_{k}-y\right\|^{2}+\left\|y_{k}-y\right\|^{2} \leqslant 2\left\|x_{k}-y\right\|^{2}+\widetilde{\theta}_{k} .
$$

Hence

$$
\begin{aligned}
\left\|z_{k}-z\right\|^{2}+\left\|y_{k}-z\right\|^{2}= & \left\|z_{k}-(\alpha x+(1-\alpha) y)\right\|^{2}+\left\|y_{k}-(\alpha x+(1-\alpha) y)\right\|^{2} \\
= & \left\|\alpha\left(z_{k}-x\right)+(1-\alpha)\left(z_{k}-y\right)\right\|^{2}+\left\|\alpha\left(y_{k}-x\right)+(1-\alpha)\left(y_{k}-y\right)\right\|^{2} \\
= & \alpha\left\|z_{k}-x\right\|^{2}+(1-\alpha)\left\|z_{k}-y\right\|^{2}-\alpha(1-\alpha)\|x-y\|^{2} \\
& +\alpha\left\|y_{k}-x\right\|^{2}+(1-\alpha)\left\|y_{k}-y\right\|^{2}-\alpha(1-\alpha)\|x-y\|^{2}
\end{aligned}
$$




$$
\begin{aligned}
= & \alpha\left(\left\|z_{k}-x\right\|^{2}+\left\|y_{k}-x\right\|^{2}\right)+(1-\alpha)\left(\left\|z_{k}-y\right\|^{2}+\left\|y_{k}-y\right\|^{2}\right) \\
& -2 \alpha(1-\alpha)\|x-y\|^{2} \\
\leqslant & \alpha\left(2\left\|x_{k}-x\right\|^{2}+\widetilde{\theta}_{k}\right)+(1-\alpha)\left(2\left\|x_{k}-y\right\|^{2}+\widetilde{\theta}_{k}\right)-2 \alpha(1-\alpha)\|x-y\|^{2} \\
= & 2\left[\alpha\left\|x_{k}-x\right\|^{2}+(1-\alpha)\left\|x_{k}-y\right\|^{2}-\alpha(1-\alpha)\|x-y\|^{2}\right]+\alpha \widetilde{\theta}_{k}+(1-\alpha) \widetilde{\theta}_{k} \\
= & 2\left\|\alpha\left(x_{k}-x\right)+(1-\alpha)\left(x_{k}-y\right)\right\|^{2}+\widetilde{\theta}_{k} \\
= & 2\left\|x_{k}-(\alpha x+(1-\alpha) y)\right\|^{2} \\
= & 2\left\|x_{k}-z\right\|^{2}+\widetilde{\theta}_{k} .
\end{aligned}
$$

It follows that $z \in C_{k+1}$ and hence $C_{k+1}$ is convex. Therefore, $C_{n}$ is closed and convex for all $n \in \mathbb{N}$. This implies that $\left\{x_{n}\right\}$ is well-defined. Since $x_{n}=P_{C_{n}} x_{0}$, it follows that

$$
\left\langle x_{0}-x_{n}, x_{n}-y\right\rangle \geqslant 0,
$$

for all $y \in F(T) \cap F(S)$ and $n \in \mathbb{N}$. So $u \in F(T) \cap F(S)$, we have

$$
\begin{aligned}
0 & \leqslant\left\langle x_{0}-x_{n}, x_{n}-u\right\rangle=-\left\langle x_{n}-x_{0}, x_{n}-x_{0}\right\rangle+\left\langle x_{0}-x_{n}, x_{0}-u\right\rangle \\
& \leqslant-\left\|x_{n}-x_{0}\right\|^{2}+\left\|x_{0}-x_{n}\right\|\left\|x_{0}-u\right\| .
\end{aligned}
$$

This implies that

$$
\left\|x_{0}-x_{n}\right\|^{2} \leqslant\left\|x_{0}-x_{n}\right\|\left\|x_{0}-u\right\|,
$$

and hence

$$
\left\|x_{0}-x_{n}\right\| \leqslant\left\|x_{0}-u\right\|,
$$

for all $u \in F(T) \cap F(S)$ and $n \in \mathbb{N}$. From $x_{n}=P_{C_{n}} x_{0}$ and $x_{n+1}=P_{C_{n+1}} x_{0} \in C_{n+1} \subseteq C_{n}$, we obtain that

$$
\left\langle x_{0}-x_{n}, x_{n}-x_{n+1}\right\rangle \geqslant 0,
$$

for all $n \in \mathbb{N}$. So, for all $x_{n+1} \in C_{n+1}$, for $n \in \mathbb{N}$, we have

$$
\begin{aligned}
0 & \leqslant\left\langle x_{0}-x_{n}, x_{n}-x_{n+1}\right\rangle=-\left\langle x_{n}-x_{0}, x_{n}-x_{0}\right\rangle+\left\langle x_{0}-x_{n}, x_{0}-x_{n+1}\right\rangle \\
& \leqslant-\left\|x_{n}-x_{0}\right\|^{2}+\left\|x_{0}-x_{n}\right\|\left\|x_{0}-x_{n+1}\right\| .
\end{aligned}
$$

This implies that

$$
\left\|x_{0}-x_{n}\right\|^{2} \leqslant\left\|x_{0}-x_{n}\right\|\left\|x_{0}-x_{n+1}\right\|,
$$

and hence

$$
\left\|x_{0}-x_{n}\right\| \leqslant\left\|x_{0}-x_{n+1}\right\|,
$$

for all $n \in \mathbb{N}$. Since $\left\{\left\|x_{0}-x_{n}\right\|\right\}$ is bounded, $\lim _{n \rightarrow \infty}\left\|x_{n}-x_{0}\right\|$ exists. Next, we claim that

$$
\lim _{n \rightarrow \infty}\left\|x_{n}-x_{n+1}\right\|=0 \text {. }
$$

From (3.7), we have

$$
\begin{aligned}
\left\|x_{n}-x_{n+1}\right\|^{2} & =\left\|\left(x_{n}-x_{0}\right)+\left(x_{0}-x_{n+1}\right)\right\|^{2} \\
& =\left\|x_{n}-x_{0}\right\|^{2}+2\left\langle x_{n}-x_{0}, x_{0}-x_{n+1}\right\rangle+\left\|x_{0}-x_{n+1}\right\|^{2} \\
& =\left\|x_{n}-x_{0}\right\|^{2}-2\left\langle x_{0}-x_{n}, x_{0}-x_{n}\right\rangle-2\left\langle x_{0}-x_{n}, x_{n}-x_{n+1}\right\rangle+\left\|x_{0}-x_{n+1}\right\|^{2} \\
& \leqslant\left\|x_{n}-x_{0}\right\|^{2}-2\left\|x_{n}-x_{0}\right\|^{2}+\left\|x_{0}-x_{n+1}\right\|^{2} \\
& =-\left\|x_{n}-x_{0}\right\|^{2}+\left\|x_{0}-x_{n+1}\right\|^{2} .
\end{aligned}
$$


Since, $\lim _{n \rightarrow \infty}\left\|x_{n}-x_{0}\right\|$ exists, we have $\lim _{n \rightarrow \infty}\left\|x_{n}-x_{n+1}\right\|=0$. Since $x_{n+1} \in C_{n}$, we have

$$
\left\|z_{n}-x_{n+1}\right\|^{2}+\left\|y_{n}-x_{n+1}\right\|^{2} \leqslant 2\left\|x_{n}-x_{n+1}\right\|^{2}+\widetilde{\theta}_{n}
$$

From $\lim _{n \rightarrow \infty}\left\|x_{n}-x_{n+1}\right\|=0$ and $\theta_{n} \rightarrow 0$ as $n \rightarrow \infty$, it follows that

$$
\lim _{n \rightarrow \infty}\left\|z_{n}-x_{n+1}\right\|=0=\lim _{n \rightarrow \infty}\left\|y_{n}-x_{n+1}\right\|,
$$

which yields

$$
\left\|z_{n}-x_{n}\right\| \leqslant\left\|z_{n}-x_{n+1}\right\|+\left\|x_{n+1}-x_{n}\right\| \rightarrow 0, \quad \text { as } n \rightarrow \infty,
$$

and then we have

$$
\left\|y_{n}-x_{n}\right\| \leqslant\left\|y_{n}-x_{n+1}\right\|+\left\|x_{n+1}-x_{n}\right\| \rightarrow 0, \quad \text { as } n \rightarrow \infty .
$$

We now claim that

$$
\limsup _{r \rightarrow \infty} \limsup _{n \rightarrow \infty}\left\|T(r) x_{n}-x_{n}\right\|=0=\limsup _{r \rightarrow \infty} \limsup _{n \rightarrow \infty}\left\|S(r) x_{n}-x_{n}\right\| .
$$

Indeed, by definition of $y_{n}$ and $x_{n+1} \subset C_{n}$ we have

$$
\left\|\frac{1}{t_{n}} \int_{0}^{t_{n}} T(t) x_{n} d t-x_{n}\right\|=\frac{1}{1-\alpha_{n}}\left\|y_{n}-x_{n}\right\| \rightarrow 0, \quad \text { as } n \rightarrow \infty .
$$

From $z_{n}=\beta_{n}\left[\gamma_{n} y_{n}+\left(1-\gamma_{n}\right) x_{n}\right]+\left(1-\beta_{n}\right) \frac{1}{s_{n}} \int_{0}^{s_{n}} S(t) x_{n} d t$, we have

$$
\begin{aligned}
\left\|\frac{1}{s_{n}} \int_{0}^{s_{n}} S(t) y_{n} d t-z_{n}\right\| & =\frac{\beta_{n}}{1-\beta_{n}}\left\|\gamma_{n}\left(y_{n}-z_{n}\right)+\left(1-\gamma_{n}\right)\left(x_{n}-z_{n}\right)\right\| \\
& \leqslant \frac{\beta_{n}}{1-\beta_{n}}\left(\gamma_{n}\left\|y_{n}-z_{n}\right\|+\left(1-\gamma_{n}\right)\left\|x_{n}-z_{n}\right\|\right) \rightarrow 0, \quad \text { as } n \rightarrow \infty .
\end{aligned}
$$

It follows that

$$
\begin{aligned}
\left\|\frac{1}{s_{n}} \int_{0}^{s_{n}} S(t) x_{n} d t-x_{n}\right\| & \leqslant\left\|\frac{1}{s_{n}} \int_{0}^{s_{n}} S(t) x_{n} d t-\frac{1}{s_{n}} \int_{0}^{s_{n}} S(t) y_{n} d t\right\|+\left\|\frac{1}{s_{n}} \int_{0}^{s_{n}} S(t) y_{n} d t-z_{n}\right\|+\left\|z_{n}-x_{n}\right\| \\
& \leqslant \frac{1}{s_{n}} \int_{0}^{s_{n}}\left\|S(t) x_{n}-S(t) y_{n}\right\| d t+\left\|\frac{1}{s_{n}} \int_{0}^{s_{n}} S(t) y_{n} d t-z_{n}\right\|+\left\|z_{n}-x_{n}\right\| \\
& \leqslant \frac{1}{s_{n}} \int_{0}^{s_{n}} L_{t}^{S} d t\left\|x_{n}-y_{n}\right\|+\left\|\frac{1}{s_{n}} \int_{0}^{s_{n}} S(t) y_{n} d t-z_{n}\right\|+\left\|z_{n}-x_{n}\right\| \\
& \leqslant \widetilde{s}_{n}\left\|x_{n}-y_{n}\right\|+\left\|\frac{1}{s_{n}} \int_{0}^{s_{n}} S(t) y_{n} d t-z_{n}\right\|+\left\|z_{n}-x_{n}\right\| \rightarrow 0, \quad \text { as } n \rightarrow \infty .
\end{aligned}
$$

For all $0 \leqslant r<\infty$, we note that

$$
\begin{aligned}
\left\|S(r) x_{n}-x_{n}\right\| \leqslant & \left\|S(r) x_{n}-S(r)\left(\frac{1}{s_{n}} \int_{0}^{s_{n}} S(t) x_{n} d t\right)\right\|+\left\|S(r)\left(\frac{1}{s_{n}} \int_{0}^{s_{n}} S(t) x_{n} d t\right)-\frac{1}{s_{n}} \int_{0}^{s_{n}} S(t) x_{n} d t\right\| \\
& +\left\|\frac{1}{s_{n}} \int_{0}^{s_{n}} S(t) x_{n} d t-x_{n}\right\| \\
& \leqslant\left(L_{\infty}+1\right)\left\|\frac{1}{s_{n}} \int_{0}^{s_{n}} S(t) x_{n} d t-x_{n}\right\|+\left\|S(r)\left(\frac{1}{s_{n}} \int_{0}^{s_{n}} S(t) x_{n} d t\right)-\frac{1}{s_{n}} \int_{0}^{s_{n}} S(t) x_{n} d t\right\| \\
& :=\left(L_{\infty}+1\right) A_{n}^{S}(r)+B_{n}^{S}(r),
\end{aligned}
$$

where $A_{n}^{S}:=\left\|\frac{1}{s_{n}} \int_{0}^{s_{n}} S(t) x_{n} d t-x_{n}\right\|$ and $B_{n}^{S}:=\left\|S(r)\left(\frac{1}{s_{n}} \int_{0}^{s_{n}} S(t) x_{n} d t\right)-\frac{1}{s_{n}} \int_{0}^{s_{n}} S(t) x_{n} d t\right\|$. By Lemma 2.3, we have $\limsup _{n \rightarrow \infty} A_{n}^{S}(r)=0=\limsup _{n \rightarrow \infty} B_{n}^{S}(r)$. We can deduce that for all $0 \leqslant r<\infty$,

$$
\left\|T(r) x_{n}-x_{n}\right\| \leqslant\left\|T(r) x_{n}-T(r)\left(\frac{1}{t_{n}} \int_{0}^{t_{n}} T(t) x_{n} d t\right)\right\|+\left\|T(r)\left(\frac{1}{t_{n}} \int_{0}^{t_{n}} T(t) x_{n} d t\right)-\frac{1}{t_{n}} \int_{0}^{t_{n}} T(t) x_{n} d t\right\|
$$




$$
\begin{aligned}
& +\left\|\frac{1}{t_{n}} \int_{0}^{t_{n}} T(t) x_{n} d t-x_{n}\right\| \\
\leqslant & \left(L_{\infty}+1\right)\left\|\frac{1}{t_{n}} \int_{0}^{t_{n}} T(t) x_{n} d t-x_{n}\right\|+\left\|T(r)\left(\frac{1}{t_{n}} \int_{0}^{t_{n}} T(t) x_{n} d t\right)-\frac{1}{t_{n}} \int_{0}^{t_{n}} T(t) x_{n} d t\right\| \\
:= & \left(L_{\infty}+1\right) A_{n}^{T}(r)+B_{n}^{T}(r),
\end{aligned}
$$

where $A_{n}^{\top}(r):=\left\|\frac{1}{t_{n}} \int_{0}^{t_{n}} T(t) x_{n} d t-x_{n}\right\|$ and $B_{n}^{\top}(r):=\left\|T(r)\left(\frac{1}{t_{n}} \int_{0}^{t_{n}} T(t) x_{n} d t\right)-\frac{1}{t_{n}} \int_{0}^{t_{n}} T(t) x_{n} d t\right\| . \quad B y$ Lemma 2.3, we have $\limsup _{n \rightarrow \infty} A_{n}^{\top}(r)=0=\limsup _{n \rightarrow \infty} B_{n}^{\top}(r)$. Then we obtain

$$
\limsup _{r \rightarrow \infty} \limsup _{n \rightarrow \infty}\left\|T(r) x_{n}-x_{n}\right\|=0=\limsup _{r \rightarrow \infty} \limsup _{n \rightarrow \infty}\left\|S(r) x_{n}-x_{n}\right\| \text {. }
$$

We note by Lemma 2.2, that every weak limit of $\left\{x_{n}\right\}$ is a member of $\mathfrak{F}$. From $x_{n} \rightarrow z \in P_{\mathfrak{F}} x_{0}$, we have $x_{0}-x_{n} \rightarrow x_{0}-z_{0}$, form $H$ satisfies the Kadec-Klee property, it follows that

$$
x_{0}-x_{n} \rightarrow x_{0}-z_{0}
$$

So, we have

$$
\left\|x_{n}-z_{0}\right\|=\left\|x_{n}-x_{0}-\left(z_{0}-z_{0}\right)\right\| \rightarrow 0, \quad \text { as } n \rightarrow \infty .
$$

Hence, $x_{n} \rightarrow z_{0}$. This completes the proof.

\section{Acknowledgment}

The author would like to thank Uttaradit Rajabhat University for financial support. Moreover, we would like to thank Prof. Dr. Somyot Plubiteng for providing valuable suggestions.

\section{References}

[1] Q.-L. Dong, S. N. He, Y. J. Cho, A new hybrid algorithm and numerical realization for two nonexpansive mappings, Fixed Point Theory Appl., 2015 (2015), 12 pages. 1, 3.2

[2] K. Goebel, W. A. Kirk, A fixed point theorem for asymptotically nonexpansive mappings, Proc. Amer. Math. Soc., 35 (1972), 171-174. 1

[3] I. Inchan, S. Plubtieng, Strong convergence theorems of hybrid methods for two asymptotically nonexpansive mappings in Hilbert spaces, Nonlinear Anal. Hybrid Syst., 2 (2008), 1125-1135. 1, 3.2

[4] S. Ishikawa, Fixed points by a new iteration method, Proc. Amer. Math. Soc., 44 (1974), 147-150. 1

[5] T.-H. Kim, H.-K. Xu, Strong convergence of modified Mann iterations for asymptotically nonexpansive mappings and semigroups, Nonlinear. Anal., 64 (2006), 1140-1152. 1, 2.2, 2.3

[6] P.-K. Lin, K.-K. Tan, H. K. Xu, Demiclosedness principle and asymptotic behavior for asymptotically nonexpansive mappings, Nonlinear. Anal., 24 (1995), 929-946. 2.4

[7] W. A. Mann, Mean value methods in iteration, Proc. Amer. Math. Soc., 4 (1953), 506-510. 1

[8] C. Martinez-Yanes, H.-K. Xu, Strong convergence of the CQ method for fixed point processes, Nonlinear Anal., 64 (2006), 2400-2411. 2.5

[9] Z. Opial, Weak convergence of the sequence of successive approximations for nonexpansive mappings, Bull. Amer. Math. Soc., 73 (1967), 591-597. 1

[10] J. Schu, Weak and strong convergence to fixed points of asymptotically nonexpansive mappings, Bull. Amer. Math. Soc., 43 (1991), 153-159.

[11] W. Takahashi, Y. Takeuchi, R. Kubota, Strong convergence theorems by hybrid methods for families of nonexpansive mappings in Hilbert spaces, J. Math. Anal. Appl., 341 (2008), 276-286. 1, 1.1, 3.2, 3.4

[12] H.-K. Xu, Strong asymptotic behavior of almost-orbits of nonlinear semigroups, Nonlinear. Anal., 46 (2001), 135-151. 1 